ery of $P$ aeruginosa was 0 days for SPA, 11 days for MDRPA, and 24 days for HRPA.

The authors concluded that the duration of empirical antipseudomonal antibiotic treatment influences selection of resistant strains of $P$ aeruginosa; the longer the duration, the broader the pattern of resistance.

FROM: Philippe E, Weiss M, Shultz JM, Yeomans F, Ehrenkranz NJ. Emergence of highly antibiotic-resistant Pseudomonas aeruginosa in relation to duration of empirical antipseudomonal antibiotic treatment. Clinical Performance and Quality Health Care 1999;7:83-87.

\section{Evaluation of an Antiseptic Triple-Lumen Catheter in an ICU}

Hanley and coinvestigators, from the Department of Epidemiology, Albany Medical Center Hospital, Albany, New York, conducted a study to evaluate a decrease in catheter-related (CR) bloodstream infection (BSI) rate in patients with antiseptic triple-lumen catheters in an ICU.

They conducted a retrospective review of surveillance records, patient medical records, laboratory and microbiological reports, and antibiotic administration records. The study included patients admitted to the ICU with triple-lumen catheters. A subset of one entry per patient was extracted from 2 years of primary BSI surveillance data. Data collection included risk factors, laboratory and microbiological data, insertion sites, and dates of all intravascular catheters present during triple-lumen catheterization.

The CR BSI rate was 5.4 and 11.3 per 1,000 catheterdays in antiseptic and nonantiseptic triple-lumen catheter groups, respectively $(P=.06)$.

By multivariate analysis using a Cox Proportional Hazards Model, the antiseptic triple-lumen catheters (chlorhexidine gluconate and silver sulfadiazine) were associated with a significant reduction in CR BSI $(P=.03)$.

The authors concluded that the use of antiseptic triple-lumen catheters may substantially reduce CR BSIs in an intensive care population and may be subsequently associated with a decrease in length of stay.

FROM: Hanley EM, Veeder A, Smith T, Drusano G, Currie E, Venezia RA. Evaluation of an antiseptic triplelumen catheter in an intensive care unit. Crit Care Med 2000;28:366-370.

\section{TB Practices in Maryland Hospitals}

In 1992 and 1993, the Maryland Hospital Association and the Maryland Department of Health and Mental Hygiene conducted two surveys of TB prevention practices in Maryland hospitals that showed poor compliance with the 1990 CDC guidelines for preventing transmission of TB in healthcare facilities. In 1997, Fuss and colleagues conducted a study to assess compliance with the CDC's guidelines in Maryland acute-care hospitals.

A written questionnaire with three components
(Infection Control, Employee Health, and Microbiology Laboratory) was mailed to 56 Maryland acute-care hospitals. Seventy-three percent of the surveys were returned. One hundred percent of responding hospitals with TB isolation rooms reported negative-pressure ventilation, six air exchanges per hour, and air exhausted to the outside or through high-efficiency particulate air filters. One hundred percent of the responding hospitals reported providing NIOSH-approved respiratory protection for healthcare workers, compared with $24 \%$ in $1992(P<.01)$. One hundred percent of the responding hospitals reported performing at least annual tuberculin skin testing, compared with $50 \%$ in $1992(P<.01)$.

The survey results demonstrate excellent compliance with the 1994 CDC recommendations for TB control in Maryland acute-care hospitals, even in those facilities determined to be at minimal to low risk for TB exposure. The proposed OSHA regulations are unlikely to reduce the risk of TB exposure to healthcare workers in Maryland acute-care hospitals further.

FROM: Fuss EP, Israel E, Baruch N, Roghmann MC. Improved tuberculosis infection control practices in Maryland acute-care hospitals. Am J Infect Control 2000;28:133-137.

\section{Prolonged Hospital Stay and Surgical-Site Infections}

The accepted standard in estimating the stay prolongation attributable to surgical-site infections (SSIs) is the matched-cohort study (MCS) method, which is associated with selection bias. The Appropriateness Evaluation Protocol (AEP) has been used to estimate stay prolongation attributable to nosocomial infections but has not been validated specifically for SSIs.

Merle and coinvestigators, from the Rouen University Hospital-Charles Nicolle, conducted a study to compare estimates of stay prolongation attributable to SSIs after digestive surgery, obtained by AEP and by MCS. Sixty-five SSIs after digestive tract surgery were analyzed by AEP and MCS. AEP stay prolongation was the number of days judged specifically appropriate for the care of SSIs. MCS stay prolongation was the difference of stay duration in SSI cases and two controls matched by age, gender, and diagnosis-related groups. Sensitivity and specificity of AEP, and agreement between both methods, were calculated.

The mean AEP stay prolongation was 3.5 days versus 7.2 days for MCS. The sensitivity of AEP was $58 \%$, and the specificity was $75 \%$. The agreement between the two methods was poor.

The authors concluded that SSIs after digestive tract surgery increased the hospital stay. Accurate estimations of a prolongation of stay will vary according to the method selected.

FROM: Merle V, Germain JM, Chamouni P, Daubert H, Froment L, Michot F, et al. Assessment of prolonged hospital stay attributable to surgical site infections using 